\title{
Signal detectability theory applied to conceptual discrimination'
}

\section{Z. JOSEPH ULEHLA, LESLIE CANGES 2 , AND FRANCES WACKWITZ ${ }^{3}$}

Signal Detectability Theory (SDT) was applied to the discrimination of the source (magazine) of two-word and fourword samples of English text. Operating characteristics obtained from ratings of individual four-word samples were well fit by an SDT theoretical curve, but two-word data were poorly fitted. Indices of signal detectability derived from rating responses, two alternative forced choice responses, and four altermative forced choice responses were found to be equivalent, thus supporting a key implication of SDT.

Although developed as a model for psychophysical detection (Green \& Swets, 1966), signal detection theory (SDT) may prove applicable to discrimination processes in general. Toward this end, the present research extends this model to a type of conceptual judgment, the discrimination of the source of short samples of English text. Two rather different magazines were used as the alternative sources. Magazine A is a "he-man" magazine, oriented to male readers and featuring adventure and sex. Magazine B is a "confessional" magazine, oriented toward female readers and featuring firstperson accounts of romantic and family involvements. Successful application of SDT to stimuli so different from those used in prior research would suggest that SDT constitutes a useful model for human discrimination in general, irrespective of the nature of the stimuli to be discriminated.

In order to evaluate the fit of SDT to the conceptual task, two types of analysis which provide much of the empirical support for SDT in psychophysics were applied to magazine discrimination data. The first type of analysis involves the shape of the operating characteristic (OC). The OC depicts how success or hit rate (HR) in identification of one stimulus covaries with error rate (ER) in the identification of the alternative stimulus as the criterion for decision is varied over its entire range. Use of an n-category rating scale forces $S$ to use n-1 decision criteria concurrently. Each of S's decision criteria yields an estimate of a point on his OC, namely, the proportion of A samples which exceed that criterion plotted against the proportion of $B$ samples which exceed that same criterion. If the HR for each successive criterion is transformed according to the normal probability function, the resulting HR $\mathrm{z}$ scores $\left(z_{H}\right)$ are implied by SDT to bear a linear relation to the similarly transformed ERs $\left(\mathrm{z}_{\mathrm{F}}\right)$ (Green \& Swets, 1966). Since the rank-order correlation is necessarily one, the degree to which this relation is linear can be represented by a product moment correlation coefficient $(r)$ computed between $z_{H}$ and $z_{F}$. Two non-independent predictions relating to OCs can thus be drawn from SDT: (a) The $r$ between $z_{H}$ and $z_{F}$ will be greater than the $r$ between HR and ER. (b) The $r$ between $z_{H}$ and $z_{F}$ will be essentially one. The first analysis tested these predictions using ratings of samples of English text.

The second analysis tested the implication of SDT that a variety of different response conditions can be employed to yield equivalent estimates of the index of signal detectability (d'). With simplifying assumptions, tables have been published embodying the theoretical relations between response probabilities and d'(Elliott, 1959). These tables were used to obtain estimates of $d^{\prime}$ from the rating task (using the most central decision criterion) and from proportion correct in two-alternative forced-choice (2AFC) and four-alternative forcedchoice (4AFC) tasks. If the $d^{\prime}$ estimates prove to be significantly different, the theory is contradicted. Subjects

Six University of Denver undergraduate students served as paid Ss.

\section{Materials}

In total, 800 two-word and 800 four-word series of consecutive words were randomly selected from recent issues of the two magazines, A and B. For each word length, the samples were used as follows: Two hundred samples from each source were combined in a random sequence to provide materials for 400 single item ratings. Two hundred 2AFC items each contained one $A$ and one B sample. One hundred $4 \mathrm{AFC}$ items each contained one $A$ and three $B$ samples; an additional 1004 AFC items each contained three $A$ samples and one $B$ sample. In the 2AFC and 4AFC tasks, position of the alternatives was randomized. All of the items were duplicated into booklets with 20 items per booklet.

\section{Procedure}

Six experimental conditions were employed; singlesample ratings, $2 \mathrm{AFC}$, and $4 \mathrm{AFC}$ tasks using each of the two word series lengths. In the ratings condition, $S$ indicated his judgment of the source, magazine A or magazine $B$, and also indicated high, moderate, or low confidence in his judgment. Judgments for which high confidence was expressed won $1-1 / 2$ cent for $S$ when correct, lost 1-1/2 cent when incorrect. Moderate confidence and low confidence judgments were worth \pm 1 cent and $\pm 1 / 2$ cent respectively. In the $2 \mathrm{AFC}$ condition, $\mathrm{S}$ was paid 1 cent for each correct discrimination, fined 1 cent for each error. In the $4 \mathrm{AFC}$ condition, $\mathrm{S}$ indicated his first, second, and third choices as to which word series was sampled from the "single source." The $S$ was paid 2 cents if his first choice was correct, was fined 1 cent if his third choice was correct, and was fined 2 cents if the single source sample was not included 
among his three choices. The payoffs were added to S's base wage of 1 dollar per hour. Three Ss performed in the four-word conditions first; the other three Ss performed in the two-word conditions first. The materials were separated into 20 -item blocks. Within each word series length, blocks from all response conditions were mixed and presented in a different random order to each S. Upon completing each block, S checked his performance using a key furnished by $E$.

Results

One $S$ failed to distribute his responses over the different rating categories and was eliminated from the $O C$ analysis. The two-word data failed to support either of the OC predictions drawn from SDT; the rs between $\mathrm{z}_{\mathrm{H}}$ and $\mathrm{z}_{\mathrm{F}}$ were below one and were not consistently higher than the rs between the HR and ER (see Table 1). The four-word data, however, supported both predictions. Rounding to two decimals, the $z$-score rs of one predicted by SDT emerged for four of the five Ss and the fifth $S$ yielded an $r$ of .99. All of these values exceed the $r$ between HR and ER.

The $d^{\prime}$ values estimated from the various response conditions are presented in Table 2 . An analysis of variance yielded no significant effect of response condition upon $d^{\prime}(F=1.12, d f=2 / 10, p>.05)$, and no significant interaction between response condition and length of word series $(F=2.10, d f=2 / 10, p>.05)$. As expected, word series length yielded a significant main effect ( $F=78.39$, df $=1 / 10, p<.01$ ). Thus, the results of the analysis of variance are consistent with SDT.

To provide a further test for a response condition effect, each condition was divided into two arbitrary halves. Estimates of ' ' were computed for each half of each condition. Mean discrepancies of two types were then computed: (a) The discrepancy between the two halves of the same response condition were averaged within $S$ and within word series length. (b) Discrepancies between estimates of $d^{\prime}$ based on different response conditions were averaged within $S$, within word series length, and within arbitrary half. Both those discrepancies would reflect only sampling error if SDT is valid;

Table 1.

Correlation Coefficients Relating to Operating Characteristics

\begin{tabular}{ccc} 
Subject Number & $\begin{array}{r}r \text { between }{ }^{2} \mathrm{H} \text { and } \mathrm{z}_{\mathrm{F}} \\
\text { Two Word Magazine ltems }\end{array}$ & $\begin{array}{c}r \text { between } \\
H R \text { and ER }\end{array}$ \\
\hline 1 & 1.00 & .97 \\
2 & .99 & .97 \\
3 & .99 & .97 \\
4 & .98 & .99 \\
5 & .97 & .99 \\
\hline & Four Word Magazine Items & \\
1 & 1.00 & .95 \\
2 & 1.00 & .93 \\
3 & 1.00 & .92 \\
4 & 1.00 & .89 \\
5 & .99 & .93 \\
\hline
\end{tabular}

Table 2. The d' Values Estimated from Three Modes of Response for Each Word Series Length

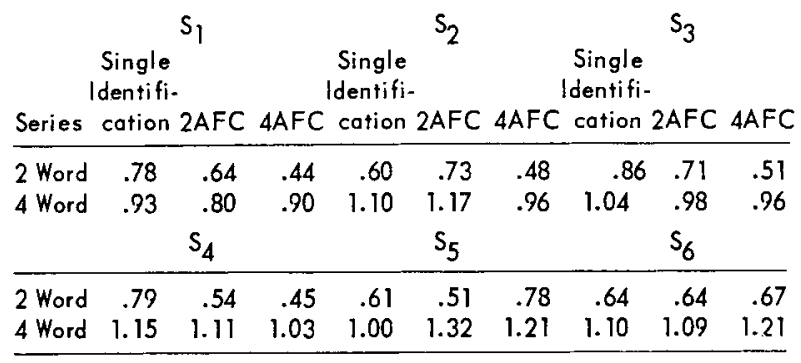

on the other hand, if response condition makes a difference, contrary to SDT, discrepancy $B$ would reflect a response condition effect in addition to sampling variance. Mean discrepancy $A$ was .20; mean discrepancy $B$ was .18. Thus, mean discrepancy $B$, which would reflect any response condition effect as well as sampling variance, was not greater than mean discrepancy $\mathrm{A}$, which reflects only sampling variance.

\section{Discussion}

The applicability of SDT to the conceptual task employed here was supported by the equivalence of $d^{\prime}$ estimates obtained from the different response conditions and by the linear relation between $z_{H}$ and $z_{F}$ yielded by the $\mathrm{OC}$ analysis in the four-word rating condition. The failure of the two-word $r^{\prime} s$ to follow the predictions may relate to the poorer discriminability of two-word samples (see Table 2). The predicted superiority of the $\mathbf{r}$ between $z_{H}$ and $z_{F}$ to the $\mathbf{r}$ between $H R$ and ER approaches zero as $\mathrm{d}^{\prime}$ approaches zero.

If further research supports the generality of SDT, wider use of such methodological values of SDT as the separation of response bias from discriminability and the convergent measure of discriminability would be appropriate. The use of threshold measures in areas where SDT applies can lead to spurious conclusions (Clark, 1966; Price, 1966).

\section{Reterences}

Clark, W. C. The psyche in psychophysics: A sensory-decision theory analysis of the effect of instructions on flicker sensitivity and response bias. Psychol. Bull., 1966, 65, 358-366.

Elliott, P. B. Tables of $d^{\prime}$. Reprinted in J. A. Swets (Ed.), Signal detection and recognition by human observers: Contemporary readings. New York: Wiley, 1964.

Green, D. M., \& Swets, J. A. Signal detcction thcory and psychophysics. New York: Wiley, 1966.

Price, R. H. Signal-detection methods in personality and perception. Psychol. Bull., 1966, 66, 55-62.

\section{Notes}

1. This research was supported by the National Science Foundation Grant GB-3081. Thanks are due to Drs. Kenneth B. Little and Donald Stilson for valuable suggestions and to Terry C. Weyl for assistance in data analysis.

2. Now at the University of Colorado.

3. Now at the University of Illinois. 